\title{
Probability of a Solution to the Solar Neutrino Problem Within the Minimal Standard Model
}

\author{
Karsten M. Heeger and R.G.H. Robertson
}

Nuclear Physics Laboratory, University of Washington, Seattle, WA 98195, U.S.A.

Tests, independent of any solar model, can be made of whether solar neutrino experiments are consistent with the minimal Standard Model (stable, massless neutrinos). If the experimental uncertainties are correctly estimated and the sun is generating energy by light-element fusion in quasi-static equilibrium, the probability of a standard-physics solution is less than $2 \%$. Even when the luminosity constraint is abandoned, the probability is not more than $4 \%$. The sensitivity of the conclusions to input parameters is explored.

12.15.Ff, 26.65.+t, 96.60.-j

The sun is believed to generate its energy by fusion reactions that can be summarized as

$$
4 \mathrm{p}+2 \mathrm{e}^{-} \rightarrow{ }^{4} \mathrm{He}+2 \nu_{e}+26.731 \mathrm{MeV} .
$$

A number of pathways lead to ${ }^{4} \mathrm{He}$, and a complex spectrum of neutrinos from $p p, p e p,{ }^{7} \mathrm{Be}$, hep $,{ }^{13} \mathrm{~N},{ }^{15} \mathrm{O},{ }^{17} \mathrm{~F}$, and ${ }^{8} \mathrm{~B}$ results [1]. The spectral shape of each individual component, whether line or continuum, is determined by laboratory measurement and/or electroweak theory. Its relative intensity, on the other hand, depends delicately on astrophysical models of the sun. The fact that these models predicted to within a factor of two the intensity of a $0.01 \%$ branch $\left({ }^{8} \mathrm{~B}\right)$ that varies as the 25 th power [2] of the central temperature of the sun must be regarded as a stunning achievement and a clear indication of the basic correctness of our understanding of how the sun and other stars function.

Nonetheless, the lack of perfect agreement raised speculation about possible exotic origins, such as neutrino oscillations. At first, the model dependence of the ${ }^{8} \mathrm{~B}$ flux calculation made such speculations interesting but not compelling. Now, however, steadily improving data from 4 independent experiments are available. The Homestake Cl-Ar experiment [3] gives $2.55 \pm 0.17 \pm 0.18$ solar neutrino units (SNU), and the Kamiokande [- 1$]$ result (increased $2 \%$ by radiative corrections [5]) is $\left(2.95_{-0.21}^{+0.22} \pm\right.$ $0.36) \times 10^{6}{ }^{8} \mathrm{~B} \nu_{e} \mathrm{~cm}^{-2} \mathrm{~s}^{-1}$. For the SAGE [6] and Gallex [7] experiments, a weighted average of $73.8 \pm 7.8 \mathrm{SNU}$ is adopted [8]. (1 $\mathrm{SNU}=10^{-36}$ events per atom per second.)
Because the three types of experiment have different energy thresholds, a coarse neutrino spectroscopy of the sun has been made. The least model-dependent questions that can be asked are,

Is it possible to describe the neutrino spectrum with any combination of the known sources in hydrogen-burning?

Is the total neutrino flux consistent with the solar luminosity?

Many have considered model-independent analyses [9 17]; in particular, Hata et al. [13] showed the data to be inconsistent with hydrogen burning and the luminosity constraint without new physics. To this body of analysis we add (a) a test of consistency free of the luminosity constraint, (b) a test for inconsistency of the data with the total solar luminosity, (c) the probabilities that the existing data would be obtained from true values in the physical regime in the absence of new physics, and (d) the dependence of the conclusions on the neutrino cross sections.

The spectral shape and endpoint of the neutrino data from Kamiokande show that ${ }^{8} \mathrm{~B}$ neutrinos are emitted from the sun and that hep neutrinos are, as expected, negligible. The pep reaction rate we take to be a fixed fraction, $f_{p e p}=0.23(2) \%$, of the $p p$ rate, [18.19] (while in principle model-dependent, $f_{p e p}$ is one of the most reliably determined model parameters, depending chiefly on the electron density and only weakly on temperature and on nuclear wavefunctions [1]). The ${ }^{7} \mathrm{Be}$ and $\mathrm{CNO}$ fluxes play a qualitatively interchangeable role in the existing experiments - the $\mathrm{Cl}-\mathrm{Ar}$ and $\mathrm{Ga}$ experiments are sensitive to both and Kamiokande to neither. As a result, it is possible to draw very general conclusions without knowledge of the relative sizes of each.

Defining the $p p+p e p,{ }^{7} \mathrm{Be}+\mathrm{CNO}$, and ${ }^{8} \mathrm{~B}$ fluxes as $\Phi_{1}, \Phi_{7+}$, and $\Phi_{8}$, respectively, the experimental capture rates as $R_{C l}$ for Cl-Ar and $R_{G a}$ for Ga-Ge, and the experimental ${ }^{8} \mathrm{~B}$ flux from Kamiokande as $R_{K a m}$, the following equations result:

$$
\begin{aligned}
a_{C 1} \Phi_{1}+a_{C 7} \Phi_{7+}+a_{C 8} \Phi_{8} & =R_{C l} \\
a_{K 8} \Phi_{8} & =R_{K a m}
\end{aligned}
$$




$$
a_{G 1} \Phi_{1}+a_{G 7} \Phi_{7+}+a_{G 8} \Phi_{8}=R_{G a}
$$

The coefficients, with the neutrino physics of the minimal Standard Model (MSM), are listed in Table 1 . The parameter $f_{C N O}$ is the fraction of the flux $\Phi_{7+}$ that is due to CNO reactions $\left(0 \leq f_{C N O} \leq 1\right)$. In Table II are shown the values of the fluxes obtained by propagating the uncertainties in the cross sections and solving. One finds that $\Phi_{7+}$ is always negative, at the same confidence level, irrespective of the value of $f_{C N O}$.

A negative flux is unphysical. Remarkably, the initial premise that the data can be described as the sum of $p p+p e p,{ }^{7} \mathrm{Be}, \mathrm{CNO}$, and ${ }^{8} \mathrm{~B}$ electron-neutrino spectra, in any proportions whatsoever, fails at the $96 \%$ confidence level 25].

A fourth neutrino-flux relationship is contained in the total solar luminosity, for a quasi-static sun deriving its energy entirely from hydrogen burning. When neutrino losses are accounted for, the electromagnetic solar constant (irradiance) $I$ in $10^{10} \mathrm{MeV} \mathrm{cm}^{-2} \mathrm{~s}^{-1}$ is given by:

$$
\begin{array}{r}
0.980\left(1-0.088 f_{\text {pep }}\right) \Phi_{1}+0.939\left(1-0.003 f_{C N O}\right) \Phi_{7+} \\
+0.498 \Phi_{8}=\frac{2 I}{Q}
\end{array}
$$

Experimentally, $I=85.31(34)$ 18, and $\mathrm{Q}=26.731$ $\mathrm{MeV}$. Additional flux constraints for hydrogen burning are given by Bahcall and Krastev [26].

Under the assumption of hydrogen burning, Eq. (1-3) can be recast with variables $I, \Phi_{7+}$, and $\Phi_{8}$ (for example). The irradiance is found to be 101(18), in agreement with the experimental value, but, as before, $\Phi_{7+}=$ $0.43(24) \times 10^{10} \mathrm{~cm}^{-2} \mathrm{~s}^{-1}$. On the other hand, forcing $\Phi_{7+}$ to zero yields $I=72(8)$, and $\chi^{2}=3.2$ for 1 degree of freedom. (Principally it is the gallium experiments that induce the strong negative correlation between the irradiance and $\Phi_{7+}$.) Thus, while any MSM solution is relatively improbable, the solar neutrino problem is not necessarily manifest in the total neutrino flux.

Including the photometrically measured luminosity as a fourth constraint reduces the uncertainties in the derived fluxes, as summarized in Table II. The probability of this result being obtained from a physically-realizable set of fluxes (i.e. with the ${ }^{7} \mathrm{Be}+\mathrm{CNO}$ flux being nonnegative) is less than $2 \%$, and quantifies directly, for example, the "last hope" suggested by Berezinsky et al. [27.

The luminosity constraint, Eq. (4), defines a plane in $\Phi_{1} \Phi_{7+} \Phi_{8}$ space. Solutions allowed in the MSM must fall within the triangular region of this plane in the positive octant (Fig. 1). The data do not meet this condition.
The assumptions made in reaching this conclusion do not include any features of solar models (one 18 is shown, for reference, in Table III). Therefore, the shape of the ${ }^{8} \mathrm{~B}$ spectrum is not as expected, containing more strength at high energies and less at low [28], and/or the neutrino flavor content is not pure electron, which alters the relationship between the Kamiokande result and the radiochemical experiments (because Kamiokande detects, via the neutral-current interaction, neutrinos of all active flavors). These features are characteristic of neutrino-oscillation solutions [9,29] 31]. In contrast to the standard-physics solution, such solutions give an excellent account of all data. Once such solutions are admitted, the fluxes may in general be quite different [32, 33.

While no astrophysical model inputs have been used in the analysis, the conclusions do depend on both neutrino cross sections and experimental uncertainties (statistical and systematic). The dependences serve to highlight the most critical experimental inputs, and aid in planning future experimental work. In Table [III the differential coefficients for the ${ }^{7} \mathrm{Be}+\mathrm{CNO}$ flux $\Phi_{7+}$ are tabulated.

Although it is a common perception that the solar neutrino problem stands or falls on the validity of the $\mathrm{Cl}-\mathrm{Ar}$ experiment, the Kamiokande datum is twice as critical. By 'critical' is meant the number of standard deviations change in an experimental result to produce a given change in $\Phi_{7+}$, i.e. the value of $\frac{\partial \Phi_{7+}}{\partial R} \sigma_{R}$.

The Ga data are almost irrelevant in the determination of the ${ }^{7} \mathrm{Be}+\mathrm{CNO}$ flux when the luminosity is a free parameter, but dominate it when the luminosity is input. This sensitivity draws attention to the importance of the neutrino cross sections, $a_{G 7}$ and $a_{G 8}$, which are determined in part by $(\mathrm{p}, \mathrm{n})$ reactions to excited states, with uncertainties that are difficult to assess. Hata and Haxton 24] have pointed out that the Gallex 34] and SAGE [6] ${ }^{51} \mathrm{Cr}$ source calibration experiments are, in fact, experimental confirmation that $a_{G 7}$ is close to the expected value unless a novel effect has caused the extraction efficiency to be low, and $a_{G 7}$ is correspondingly larger than expected. In the latter case, the calibration data make the detector response to ${ }^{7} \mathrm{Be}$ neutrinos virtually independent of the efficiency, while the response to $p p$ and ${ }^{8} \mathrm{~B}$ neutrinos scales linearly with the efficiency. The efficiency of Gallex and SAGE would both have to be reduced to $77 \%$ of the measured values to bring the derived ${ }^{7} \mathrm{Be}+\mathrm{CNO}$ flux up to zero, at which point $\chi^{2}$ exceeds 4 .

Could the present situation reflect an experimental 
result outside its estimated uncertainty? Luminosityconstrained fits of the three types of experiment in pairs give for $\Phi_{7+}$ the following values $\left(10^{10} \mathrm{~cm}^{-2} \mathrm{~s}^{-1}\right)$ : Kamiokande-Cl-Ar, -0.39(22); Gallium-Cl-Ar, -0.18(12); Kamiokande-Gallium, -0.19(11). The anomaly emerges from all combinations of pairs of experiment. This fact has the corollary that, since Gallium and $\mathrm{Cl}$-Ar have no neutral-current sensitivity, a non-standard ${ }^{8} \mathrm{~B}$ spectrum shape is somewhat favored. Experimental uncertainties in this shape contribute about $2 \%$ [35] to the error in $a_{C 8}$ and somewhat more [28] to that in $R_{K a m}$, but in a correlated way that diminishes the effect on $\Phi_{7+}$. New laboratory determinations of the spectrum are highly desirable.

At an interesting level of confidence (about 98\%), there exists a solar neutrino problem independent of solar models, except for the assumptions of neutrino production by light elements and a steady-state sun. Moreover, even abandoning the steady-state sun assumption (or, equivalently, postulating exotic energy sources) does not deliver a satisfactory solution at the $96 \%$ confidence level. With unpublished new data [36] these confidence levels reach $99.5 \%$ and $94 \%$, respectively. The numbers quantify the minimum extent of the problem in the sense that neither the ${ }^{7} \mathrm{Be}$ nor the $\mathrm{CNO}$ flux can actually be exactly zero. At the present level of significance, the data suggest new neutrino physics, and, at the same level, demonstrate that the solution to the solar neutrino problem is not to be found in the realm of astrophysics. While we keenly await results from the new generation of experiments [37, SuperKamiokande, SNO, and Borexino, we emphasize that, in this approach, there is also much to be gained from improvements to existing experiments. To illustrate the potential, setting to zero the statistical errors in the present experiments gives a result incompatible with standard physics at $>99.998 \%$ confidence level. On the other hand, systematic uncertainties are notoriously difficult to estimate, and caution is advisable. We also underscore the value of experimental work on the important cross sections $a_{C 8}, a_{G 7}$, and $a_{G 8}$, and the shape of the ${ }^{8} \mathrm{~B}$ spectrum, in the task of clarifying this fundamentally important question.

We have benefited greatly from discussions with J.N. Bahcall, S. Brice, N. Hata, W. Haxton, and J.F. Wilkerson. A. Cumming kindly corrected a number of errors. This work was supported by the U.S. Department of Energy under Grant No. DE-FG06-90ER40537.
[1] J.N. Bahcall, Neutrino Astrophysics (Cambridge 1989).

[2] J.N. Bahcall and A. Ulmer, Phys. Rev. D 53, 4202 (1996).

[3] B.T. Cleveland et al., Nucl. Phys. B (Proc. Suppl.) 38, 47 (1995).

[4] Y. Suzuki, Nucl. Phys. B (Proc. Suppl.) 38, 54 (1995).

[5] J.N. Bahcall, M. Kamionkowski, and A. Sirlin, Phys. Rev. D 51, 6146 (1995).

[6] S.R. Elliott et al., Proc. IV Intern. Workshop on Theoretical and Phenomenological Aspects of Underground Physics, Toledo, Spain, September 17-21, 1995 (to be published); J.N. Abdurashitov et al., Phys. Lett. B328, 234 (1994).

[7] Gallex Collaboration, P. Anselmann et al., Phys. Lett. B357, 237 (1995); ibid., submitted to Phys. Lett. B, July 1996.

[8] The data are assumed to be normally distributed, although there is some evidence that the distributions are not exactly normal from the asymmetric errors quoted by the experimental groups.

[9] N. Hata and P. Langacker, Phys. Rev. D 50, 632 (1994).

[10] J.N. Bahcall, Phys. Lett. B338, 276 (1994).

[11] S. Parke, Phys. Rev. Lett. 74, 839 (1995).

[12] X. Shi, D.N. Schramm, and D.S.P. Dearborn, Phys. Rev. D 50, 2414 (1994).

[13] N. Hata, S. Bludman, and P. Langacker, Phys. Rev. 49, 3622 (1994).

[14] N. Hata and P. Langacker, Phys. Rev. D 52, 420 (1994).

[15] E. Gates, L.M. Krauss, and M. White, Phys. Rev. D 51, 2631 (1995).

[16] V. Berezinsky, Comments Nucl. Part. Phys. 21, 249 (1994).

[17] V. Castellani et al. Phys. Rev. D 50, 4749 (1994).

[18] J.N. Bahcall and M.H. Pinsonneault, Revs. Mod. Phys. 67, 781 (1995).

[19] J.N. Bahcall, Phys. Rev. 135, B137 (1964); ibid. Nucl. Phys. B (Proc. Suppl.) 38, 98 (1995).

[20] J.N. Bahcall and R.K. Ulrich, Revs. Mod. Phys. 60, 297 (1988).

[21] J.N. Bahcall, Revs. Mod. Phys. 50, 881 (1978).

[22] W. Trinder et al., Phys. Lett. B349, 267 (1995).

[23] W. Hampel and R. Schlotz, Proc. Intern. Conf. Atomic Masses and Fundamental Constants 7, DarmstadtSeeheim (TH Darmstadt Lehrdruckerei, 1984), p. 89.

[24] N. Hata and W.C. Haxton, Phys. Lett. B353, 422 (1995).

[25] For a discussion of one-sided confidence levels, see Particle Data Group, Phys. Rev. D 54, 1 (1996).

[26] J.N. Bahcall and P.I. Krastev, Phys. Rev. D 53, 4211 (1996).

[27] V. Berezinsky, G. Fiorentini, and M. Lissia, Phys. Lett. B365, 185 (1996).

[28] L. deBraeckeleer, private communication.

[29] S.A.Bludman et al., Phys. Rev. D 47, 2220 (1993).

[30] P.I. Krastev and S.T. Petcov, Phys. Rev. Lett. 72, 1960 (1994); ibid Phys. Rev. D 53, 1665 (1996).

[31] G. Fiorentini et al., Phys. Rev. D 49, 6298 (1994).

[32] W. Kwong and S.P. Rosen, Phys. Rev. Lett. 73, 369 
(1994)

[33] J.N. Bahcall, M. Fukugita, and P.I. Krastev, Phys. Lett. B374, 1 (1996).

[34] Gallex Collaboration, P. Anselmann et al. Phys. Lett. B342, 440 (1995).

[35] J.N. Bahcall, E. Lisi, D.E. Alburger, L. deBraeckeleer, S.J. Freedman, and J. Napolitano, Phys. Rev. C 54, 411 (1996).

[36] V. Gavrin; T. Kirsten; K. Lande; Y. Suzuki, in Proc. International Conf. on Neutrino Physics and Astrophysics "Neutrinos '96" (Helsinki, June 13-19, 1996, to be published).

[37] See, for example, Particle Physics: Perspectives and Opportunities, edited by R. Peccei et al. (World Scientific, Singapore, 1995).
FIG. 1. The luminosity plane defined by Eq. 1, and (inset) the 1.64-standard-deviation contours (95\% confidence level for $\left.\Phi_{7+}\right)$ from the data for selected values of $f_{C N O}$. The fluxes are in units of $10^{10} \mathrm{~cm}^{-2} \mathrm{~s}^{-1}$. Solutions allowed by the MSM and the luminosity constraint must fall within the triangular area. Below the dashed lines parameterized by $f_{C N O}$, the Bahcall-Krastev condition $\Phi_{1} \geq \Phi_{7}+\Phi_{8}$ required in hydrogen burning is not met. 
TABLE I. Cross-section coefficients.

\begin{tabular}{llll}
\hline \hline & & Cross Section & Reference \\
& $10^{-46} \mathrm{~cm}^{2}$ & \\
\hline \multirow{2}{*}{ Cl-Ar } & $a_{C 1}$ & $16 f_{p e p}$ & \\
& $a_{C 7}$ & $2.38\left(1+2.60 f_{C N O}\right)$ \\
& $a_{C 8}$ & 11100 & 20 \\
\hline Kamiokande & $a_{K 8}$ & $10000^{a}$ & 20 \\
\hline \multirow{2}{*}{ Gallium } & $a_{G 1}$ & $11.8\left(1+17 f_{p e p}\right)$ \\
& $a_{G 7}$ & $76.5\left(1+1.42 f_{C N O}\right)$ \\
\hline & $a_{G 8}$ & 24600 \\
\hline
\end{tabular}

TABLE II. Fitted values of the fluxes $\left(10^{10} \nu \mathrm{cm}^{-2} \mathrm{~s}^{-1}\right)\left(f_{C N O}=0, f_{p e p}=0.0023\right)$.

\begin{tabular}{llllll}
\hline \hline Component & $\begin{array}{l}\text { Value } \\
\text { (Luminosity }\end{array}$ & $\begin{array}{l}\text { Uncertainty } \\
\text { unconstrained) }\end{array}$ & $\begin{array}{l}\text { Value } \\
\text { (Luminosity constrained) }\end{array}$ & Uncertainty & SSM $^{a}$ \\
\hline$p p+p e p$ & 8.1 & 1.7 & 6.75 & 0.11 & $5.91+0.01$ \\
${ }^{7} \mathrm{Be}+\mathrm{CNO}$ & -0.43 & 0.24 & -0.25 & 0.11 & $0.52+0.12$ \\
${ }^{8} \mathrm{~B}$ & 0.00030 & 0.00004 & 0.00027 & 0.00003 & 0.00066 \\
$I$ & 101 & 18 & 85.32 & 0.34 & 85.31 \\
$\chi^{2}$ & & 0.8 & & \\
Probability & $4 \%$ & & $1.7 \%$ & & \\
${ }^{a}$ Ref. 18 & & & & \\
\hline \hline
\end{tabular}

TABLE III. Differential coefficients for the flux $\Phi_{7+}\left(10^{10} \nu\right.$ $\left.\mathrm{cm}^{-2} \mathrm{~s}^{-1}\right) .\left(f_{C N O}=0.185, f_{p e p}=0.0023\right)$

\begin{tabular}{lrrl}
\hline \hline Parameter & $\begin{array}{r}\frac{\partial \Phi_{7+}}{\partial X} X \\
\text { (Luminosity }\end{array}$ & $\begin{array}{r}\frac{\partial \Phi_{7+}}{\partial X} X \\
\text { (Luminosity }\end{array}$ & Uncertainty $\frac{\Delta X}{X}$ \\
$\mathrm{X}$ & unconstrained) & constrained) & $\%$ \\
\hline$a_{C 1}$ & -0.09 & -0.02 & 1.2 \\
$a_{C 7}$ & +0.29 & +0.02 & 1.2 \\
$a_{C 8}$ & -1.01 & -0.17 & 3 \\
$a_{G 1}$ & +0.08 & -0.73 & 1 \\
$a_{G 7}$ & -0.02 & +0.18 & 13 \\
$a_{G 8}$ & +0.01 & -0.07 & +28 \\
$R_{C l}$ & +0.79 & +0.18 & 10 \\
$R_{K a m}$ & -1.00 & -0.31 & 14 \\
$R_{G a}$ & -0.07 & +0.65 & 11 \\
$f_{\text {pep }}$ & -0.08 & -0.06 & 10 \\
$f_{C N O}$ & +0.10 & +0.04 & \\
$I$ & - & -0.72 & 0.4 \\
\hline$\Phi_{7+}=$ & $-0.29(16)$ & $-0.19(8)$ & \\
\hline \hline
\end{tabular}

